\title{
Biomimetic artificial water channels membranes for enhanced desalination
}

Maria Di Vincenzo, ${ }^{1}$ Alberto Tiraferri, ${ }^{2}$ Valentina-Elena Musteata, ${ }^{3}$ Stefan Chisca, ${ }^{3}$ Rachid Sougrat, ${ }^{4}$ Li-Bo Huang, ${ }^{1,5}$ Suzana P. Nunes, ${ }^{3}$ Mihail Barboiu ${ }^{1,5}$

${ }^{1}$ Institut Européen des Membranes, Adaptive Supramolecular, Nanosystems Group, University of Montpellier, ENSCM, CNRS, Place Eugène Bataillon, CC 047, F-34095, Montpellier, France. ${ }^{2}$ Department of Environment, Land and Infrastructure Engineering (DIATI), Politecnico di Torino, Corso Duca degli Abruzzi 24, 10129 Turin, Italy. ${ }^{3}$ King Abdullah University of Science and Technology (KAUST), Biological and Environmental Science and Engineering Division, Advanced Membranes and Porous Materials Center, 23955-6900, Thuwal, SaudiArabia. ${ }^{4}$ King Abdullah University of Science and Technology (KAUST), Core Labs, 23955-6900, Thuwal, Saudi Arabia ${ }^{5}$ Lehn Institute of Functional Materials, School of Chemistry, Sun Yat-Sen University, Guangzhou 510275, China.

*Corresponding Author. E-mail: mihail-dumitru.barboiu@umontpellier.fr

\begin{abstract}
Biological protein channels in natural membranes utilize highly selective pores to provide exceptional transport performances, in magnitudes far above those achieved by synthetic membranes used for desalination and water treatment. Inspired by biological models, artificial water channels can be used to overcome the permeability/selectivity trade-off of traditional desalination membranes. We demonstrate that rational incorporation of I-quartet artificial water channels in composite polyamide membranes synthesized via interfacial polymerization, provide the first example of biomimetic membranes with intrinsic water-to-salt permeability on the operational RO desalination pressure and high salinity conditions. The hybrid polyamide network present larger voids and seamlessly incorporates crystalline assemblies containing I-quartet water channels for highly selective transport of water, while effectively rejecting ions or molecules to enable $\AA$-scale separations. The best biomimetic composite membranes, which can be easily scaled for industrial standards $\left(>\mathrm{m}^{2}\right)$, provide remarkable $99.5 \%$ rejection of $\mathrm{NaCl}$ with water flux of $75 \mathrm{~L} \mathrm{~m}^{-2} \mathrm{~h}^{-1}$ at 65 bar applied pressure with a 35,000 ppm $\mathrm{NaCl}$ feed solution at $\mathrm{pH} \mathrm{8,} \mathrm{representative} \mathrm{of} \mathrm{seawater} \mathrm{desalination.} \mathrm{This} \mathrm{flux} \mathrm{is} \mathrm{more}$ than $75 \%$ higher than that observed with current state-of-the-art membranes with equivalent solute rejection, translating into an equivalent reduction of the membrane effective area for the same water output and a $\sim 12 \%$ reduction of the required energy for desalination.
\end{abstract}


Water scarcity is one of the most important challenges of our time. $(1,2)$ Membrane-based technologies have a tremendous role in addressing this challenge by allowing efficient water purification and desalination. (3) Desalination membranes allowing the transport of water while rejecting solutes at high rate are used in traditional reverse osmosis (RO) processes, like seawater reverse osmosis (SWRO) and brackish water reverse osmosis (BWRO).

Thin-film composite (TFC) polyamide (PA) membranes prepared via interfacial polymerization (IP), have served as standard materials for SWRO and BWRO during the last 40 years (4). The control of the film thickness and roughness $(5,6)$, the induction of Turing structures by tuning the monomer diffusion (7), or the incorporation of porous nanofillers (8-10) have been shown to strongly increase the water permeability, while generally leading to lower selectivity, as the resulting defects in the active membrane layer affect the solute retention rate. Fundamentally, to overcome the selectivitypermeance trade-off whilst keeping a water permeance at least in the range 2-4 $\mathrm{L} \mathrm{m}^{-2} \mathrm{~h}^{-1} \mathrm{bar}^{-1}(11)$, completely new approaches would be required.

Biological water channels, the Aquaporins-AQPs (12), exhibit high water permeability and virtually total ionic rejection. The correlated water-protein H-bonding interactions within the 2.8- $\AA$ constrained AQP pores stabilize a water superstructure that prevents its disruption and explain the selective salts/proton exclusion. (13) AQPs have been embedded in artificial bio-assisted membranes to enhance the water permeance. Nonetheless, there are many challenges to overcome to preserve the protein activity at high salinity and high-pressure conditions, or to obtain high-density homogeneous AQP-based membranes. (14) Parallel to these investigations, straightforward biomimetic synthetic approaches to create artificial water channels-AWC have been proposed. $(15,16)$ The development of artificial water channels (17-20) and carbon nanotube porins (21) have increased the prospect to use these materials in highly selective water desalination technologies, since their structure can be tuned to control the water recognition, self-assembly, and translocation in a biomimetic fashion. (22) The precisely designed channel superstructures are ideal to create membrane with near-perfect selectivity. (23) Centimeter scale flat-sheet polymeric membranes embedding carbon nanotubes, (24) 
graphene, (25) graphene/carbon nanotubes (26), or pillar[5]arene channels (27) have led to large improvements in water permeance $\left(20-65 \mathrm{~L} \mathrm{~m}^{-2} \mathrm{~h}^{-1} \mathrm{bar}^{-1}\right)$ compared to traditional polyamide, but the salt rejection of these films is typically limited to values of $97 \%$, positioning these membranes in the nanofiltration domain, with little interest for seawater desalination. These results are related to important challenges in translating molecular transport properties to performant meter-scale membranes, thus filtration modules needed for effective desalination. We postulate that one of the creative strategies for addressing such scale-up challenges and to achieve improved performances, in terms of both permeability and selectivity, is to combine the polyamide material, known for its scalability via the integration within a typical roll-to-roll processing system, with the highly permeable and selective artificial water channels. The key challenge in the construction of such hybrid material is the required adaptive interaction between polyamide chains and water channels, preventing the formation of defects.

Herein, we report the incorporation of homogeneously packed AWC within a polyamide (PA) matrix, resulting in the production of scalable biomimetic membranes that remarkably outperform the classical TFC polyamide membranes in the treatment of highly saline feed streams. The novel strategy was aimed to be as simple and broadly applicable as possible. This study leads to a greater fundamental understanding of how AWC incorporation, serendipitously starting from their colloidal self-assembled superstructures, can be optimized at the nanoscale to facilitate the ultrafast and highly selective transport of water, mainly occurring through channels, and to minimize the translocation of ions and molecules bypassing the membrane via defects. One novelty here is related to the identification of unexpected new sponge-like superstructures of the hybrid PA-AWC materials. Other selective channels or materials have been previously reported in literature (23-27), but the biomimetic membranes reported here are the first materials of this type tested and applied under real seawater desalination conditions. The quantification of functional biomimetic AWC is relatively straightforward. The proposed previously unreported strategy could be subsequently used to construct RO membranes in larger scale. 
The imidazole-quartet, I-quartet, i.e., stacks of four imidazoles and two water molecules that form AWC with a pore of $2.6 \AA$, very close to natural AQP pores, present water permeability in the range of $10^{6}$ water molecules $\mathrm{s}^{-1}$ per channel with a perfect salt rejection in the lipid bilayer membranes, as confirmed previously by both experimental and theoretical results. (20) They represent incipient AWC model systems of prime importance for enhancing water transport along oriented dipolar water wires. (22) Our fabrication strategy of composite I-quartet-PA layers adds one simple preliminary step to the conventional method to synthesize reverse osmosis membranes via interfacial polymerization (4) (Fig.1A): Hexylureido-imidazole monomers (HC6) were dissolved (1.5 w/w\%) in a mixed ethanol/water $(85 / 15 \mathrm{v} / \mathrm{v} \%)$ solution, followed by sonication to obtain a homogeneous solution of I-quartet AWC colloidal aggregates. This solution was poured onto the active surface of commercial polysulfone (PSf) support layers before starting the traditional IP procedure, which was the same as that used to prepare the control TFC membranes. During the polymerization, the selfassembled I-quartet channels can produce highly dispersed crystalline phases, which are probably cross-linked with growing PA segments into hybrid assemblies or nanoparticles of 30-40 nm in diameter, after a controlled aggregation / crystallization process. These nanoparticles are integrated into the PA film during the interfacial polymerization. The morphology and performance of the final layer depend on the density of the self-assembled I-quartet nanoparticles and on their interaction with the surrounding PA matrix. To improve the performance and the homogeneity the AWCs distribution, different IP protocols were investigated. In the best procedure, the I-quartet solution was left to sit on the PSf for $60 \mathrm{~s}$ and a $2 \mathrm{w} / \mathrm{w} \%$ solution of MPD monomer was made to react with a $0.1 \mathrm{w} / \mathrm{w} \%$ solution of TMC monomer during the ensuing IP; the membranes thus formed are referred to as “optimized TFC-HC6 membranes”. 

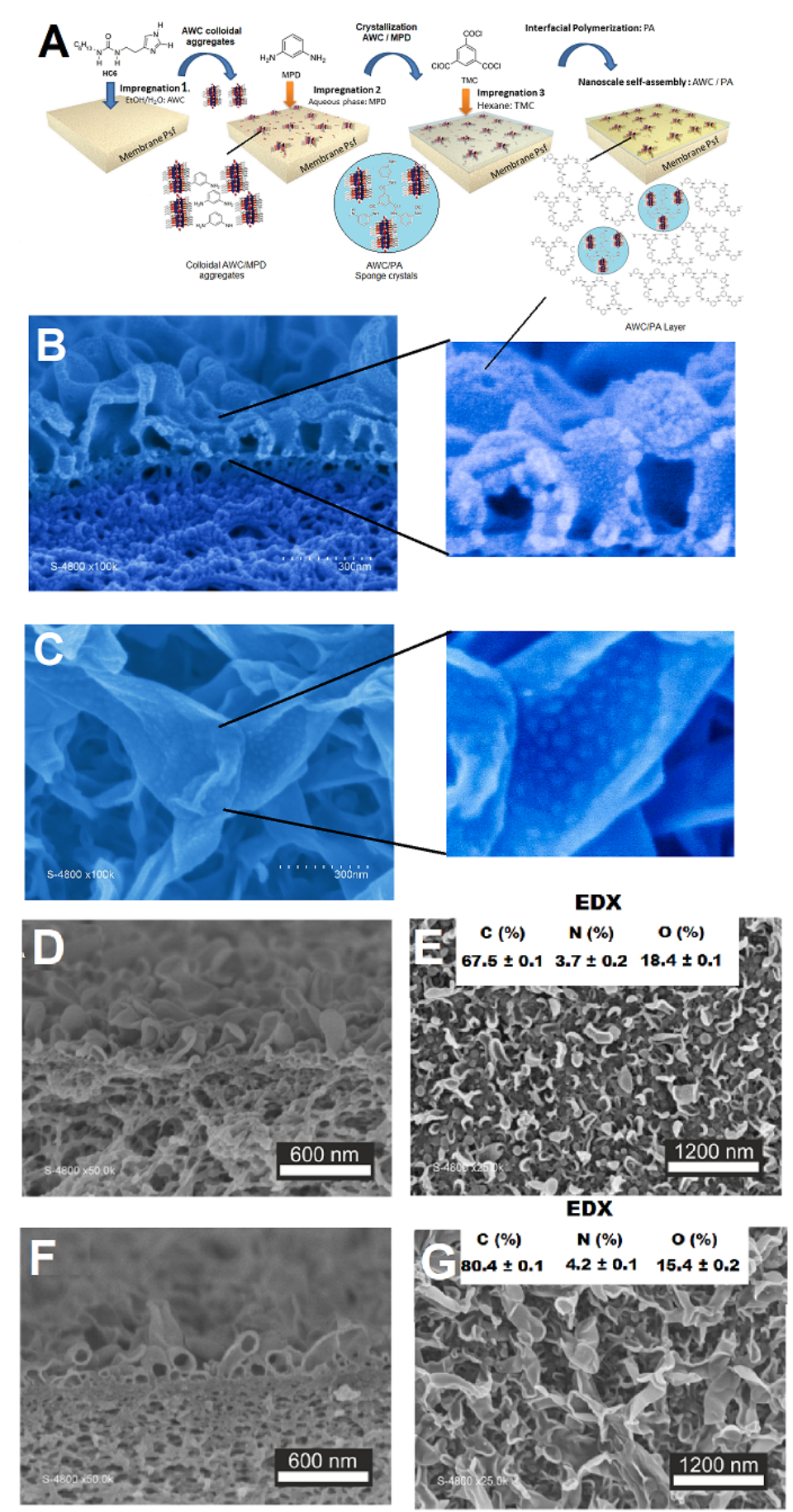

Fig. 1. Membrane preparation and Scanning Electron Microscopy (SEM) characterization (A) Synthetic procedure for the biomimetic membrane TFC-HC6 preparation: impregnation 1 of the PSf ultrafiltration support with an aqueous/ethanolic solution of artificial water channels HC6, followed by the impregnation 2 with an aqueous solution of m-phenylenediamine (MPD) monomer and then the impregnation 3 with a hexane solution of trimesoyl chloride (TMC) monomer to give rise to the interfacial polymerization (IP) and obtain the composite polyamide (PA) TFC-HC6 membranes incorporating AWCs. Representative SEM micrographs of (B) cross-sectional and (C) top view of the uppermost layer of TFC-HC6 films. SEM micrographs of $(D, F)$ cross-sectional and $(E, G)$ surface the control (D, E) TFC membranes with smaller features and (F, G) TFC-HC6 membranes with larger expanded features on PSf porous supports. The elemental analysis was performed for each membrane by using Energy Dispersive X-ray spectroscopy (EDX).

To gain insight into the mechanism of formation of AWC colloidal aggregates, we also monitored 
changes over time of different AWC samples by dynamic light scattering (DLS) experiments, before their integration into the PA layers. When $0.2 \mathrm{~mL}$ of water was added to a $1-\mathrm{mL}$ ethanolic solution of $0.5 \%$ HC6 monomer, a reversible formation of aggregates $\left(D_{h}=600-800 \mathrm{~nm}\right)$ in solution was observed, which was followed by a re-dispersion via ultra-sonication for 15 min (Fig. S2A). Small angle X-ray scattering (SAXS) profiles provided information on a lower size scale and confirmed that no structures smaller than $150 \mathrm{~nm}$ were detected for HC6 solutions with this composition (Fig. S3). For more concentrated solutions of $1.0-2.0 \% \mathrm{HC}$, smaller and more stable aggregates $\left(\mathrm{D}_{\mathrm{h}}=\right.$ $70-200 \mathrm{~nm}$ ) were observed, after adding larger amounts of water (2-6 mL water to $1 \mathrm{~mL}$ HC6 solution), without any visible precipitation (Fig.S2B,C). More interestingly, smaller aggregates $\left(\mathrm{D}_{\mathrm{h}}\right.$ $=70 \mathrm{~nm}$ ) were obtained when a $2 \mathrm{w} / \mathrm{w} \%$ aqueous solution of MPD was used instead of pure water $\left(D_{h}=200 \mathrm{~nm}\right)$, due to strong H-bonding interactions between colloidal aggregates of AWC and MPD (Table S1). Additional SAXS experiments were performed using samples with initial concentrations of $1 \%$ HC6 in 5:1 ethanol/water, after addition of water to shift the solvent mixture ratio to $1: 1$. The SAXS profiles indicate the presence of a population of mass-fractal structures with about $15 \mathrm{~nm}$ radius of gyration and Porod slope around 3 (Table S2, Fig. S4). These conditions simulate those corresponding to the best results for the nanoaggregates formation and IP membrane preparation in this study. To better explain the kinetics of the hybrid AWC-PA particles formation and integration into PA hybrid layers, it is important first to consider the nucleation process of the HC6 mass fractal colloidal structures in water/alcohol solutions, and the spontaneous nano-crystallization of selfassembled AWCs in dilute aqueous solutions. First, we verified the compatibility between the bulk X-ray fingerprint of crystalline powders obtained from colloidal suspensions, characterized by powder X-ray diffraction (PXRD) (Fig. S5C), the model lamellar packing expected for an I-quartet single crystal (Fig. S5B), and the lamellar structures imaged by Transmission Electron Microscopy (TEM) (Fig. S5A). The PXRD patterns are indicative of self-organized lamellar phases, with the main diffraction peaks at $2 \theta=3.46^{\circ}(100), 6.95^{\circ}(200)$, and $8.04^{\circ}(300)$, corresponding to interplanar distances of $25.4,12.5$, and $8.4 \AA$, respectively $(20,22)$, and of the two contracted polymorphs $(2 \theta=$ 
$3.62^{\circ}$ or $\left.4.09^{\circ}(100)\right)$, representing up to $10 \%$ of the weight of the specimen. The match between the distances of the ordered rows, observed in the TEM micrograph (Fig. S5A), and the periodicity of parallel sheets of the alternative I-quartet channels in single crystal structure (Fig. S5B), indicates that the crystalline nanoparticles obtained from colloidal solutions have the same layered arrangement.

For the membrane formation, the HC6 solution is poured on the surface of a porous PSf support. An aqueous solution containing MPD is then contacted with the HC6/PSf surface and HC6 nanoaggregated phases are formed. As observed by DLS, larger particles are formed with increasing amount of water, as HC6 is not soluble in water. During this step, a heterogeneous nucleation is promoted by H-bonding interactions between MPD and the nanoagregated water-exposed imidazoles of colloidal AWCs. After that, the membrane is immersed in the TMC organic phase. The polymerization reaction between MPD and TMC occurs very fast (roughly 10 seconds) at the organic phase side of the interface. In this step, the diffusion of the pre-formed AWC/MPD colloidal nanoparticles to the organic phase is disfavored, compared to the diffusion of the smaller MPD molecules, but they may interact with growing PA segments via H-bonding. This results in the formation of cross-linked hybrid AWC-PA nanoparticles. They are embedded into the incipient PA layer, giving rise to crystalline domains of assembled I-quartets.

The new type of PA-based hybrid material with different highly innovative inner nanostructure is therefore generated via a nucleation/interfacial PA polymerization mechanism, supported by the here reported experimental results. SEM cross-sectional (Fig. 1B) and surface (Fig. 1C) images of the TFC-HC6 membrane show the presence of the nanoparticles, incorporated and homogeneously distributed in the PA layer. The close inspection of the SEM cross-sections and membrane surfaces (Fig. 1F, G) reveals a ridge-and-valley surface morphology typical of interfacial polymerization (28, 29). However, the features observed with the incorporation of the HC6 particles are clearly larger than those of the pristine TFC membrane (Fig. 2D, E). This is also confirmed by atomic force microscopy (Fig. S6). Although several observations linking the water permeance with the 
morphology of PA layers have been reported (28-30), the exact correlation between them is still controversial (31).

The TEM microscopy confirmed that, compared to the pristine TFC layers, which have an overall more homogeneous thickness of $\sim 250 \mathrm{~nm}$ (Fig. 2A), the TFC-HC6 layers (Fig. 2B) have alternated thinner $(\sim 250 \mathrm{~nm})$ and thicker $(\sim 500 \mathrm{~nm})$ regions on the top of the porous support, a morphology that might also contribute to a higher surface area and, therefore, higher permeance. It is also clear that we have a more complex internal structure when the AWCs are added, with differentiated domains in the TFC-HC6 layers. The internal AWC/PA nanoparticles, probably corresponding to the brigher spots in Fig. 2B and S7B-D), are clearly observed also by TEM. They have diameter $\sim 20-40 \mathrm{~nm}$ and are homogeneously enclosed (30-40 spots $\left./ 100 \mu \mathrm{m}^{2}\right)$ within the PA matrix (Fig. $\left.2 \mathrm{~B}\right)$. The PA layer has been preferentially stained with $\mathrm{OsO}_{4}$ or $\mathrm{RuO}_{4}$, to improve the contrast of the PA reagions, that appears darker/grey in the regular cross-sectional TEM images of TFC (Fig. 2A) and TFC-HC6 membranes (Fig 2B). These areas are also clearly seen in the $\mathrm{RuO}_{4}$ stained 2D TEM in Fig. S7 and in the $\mathrm{RuO}_{4}$ stained TEM tomography (Fig. S8) images. In the TFC-HC6 membranes, the heavy elements (Os or $\mathrm{Ru}$, with high electron density) particularly stain the PA layer and are distributed around the brighter spots (Fig 2B, detail) with a lower electron density.

The presence of AWC components inside the membrane material is supported by Attenuated Total Reflectance (ATR)- Fourier Transform Infrared (FTIR) (Fig. S9) and Energy Dispersive X-ray (EDX) (Fig. 1E,G, Table S3) spectroscopies: a) in addition to the vibrational bands of the PA matrix present in the TFC membrane FTIR spectra of TFC-HC6 contain the $-\mathrm{CH}_{2^{-} \text {,as }}$ and $-\mathrm{CH}_{2^{-} \text {,sym }}$ stretches of the alkyl chains are visible in the range $2750-2957 \mathrm{~cm}^{-1}$ and urea-NHC $=\mathrm{O}$ and imidazole at 1612 $\mathrm{cm}^{-1}$ and $1579 \mathrm{~cm}^{-1}$ stretch suggesting the incorporation of the HC6; b) the sharp band at $3300 \mathrm{~cm}^{-1}$ attributed to the $\mathrm{O}-\mathrm{H}$ stretching vibration of strongly $\mathrm{H}$-bonded water within I-quartet channels became much broad for the TFC-HC6 membrane, due to the presence of more relaxed water within the matrix, suggesting more mobile water in the structure of hybrid TFC-HC6 membrane; c) the elemental composition determined by EDX varied when AWC were incorporated in the PA matrix, 
exhibiting a trend with up to the $10-12 \%$ higher fraction of $\% \mathrm{C}$ for TFC-HC6 layers and a concurrent reduction mainly in $\% \mathrm{O}$.

The complexity of the structure of TFC-HC6 films compared with simpler TFC films can be more clearly seen in the 3D TEM tomography movies presented as supporting information or as screen shots of the 100-nm slices of the samples (Fig. S8) while tilting them at different angles (Fig. 2C), showing that larger voids and bright spots can be observed inside the hybrid TFC-HC6 membrane when compared with the pristine TFC membrane micrographs (shown in Fig. S7A and S8A), where very few bright spots are seen. The PSf support has much less contrast, appearing as light gray areas in the regular TEM (below the yellow lined in Fig. 2A), while the PA layer formed on the surface of the PSf porous support is clearly darker. In the tomography in Fig. 2D, the position of the interface between the PSf support and the formed PA layer is represented by a dash line. The less dense white grey spots that could be mostly composed of AWC/PA nanoparticles are marked purple in Fig. 2D. The relative volume occupied by the bright (purple in Fig. 2D) spots was estimated to be $20 \%$, by conducting the segmentation and calculating the total voxel of the purple zones relative to the total volume occupied by the complete interfacially polymerized layer. 

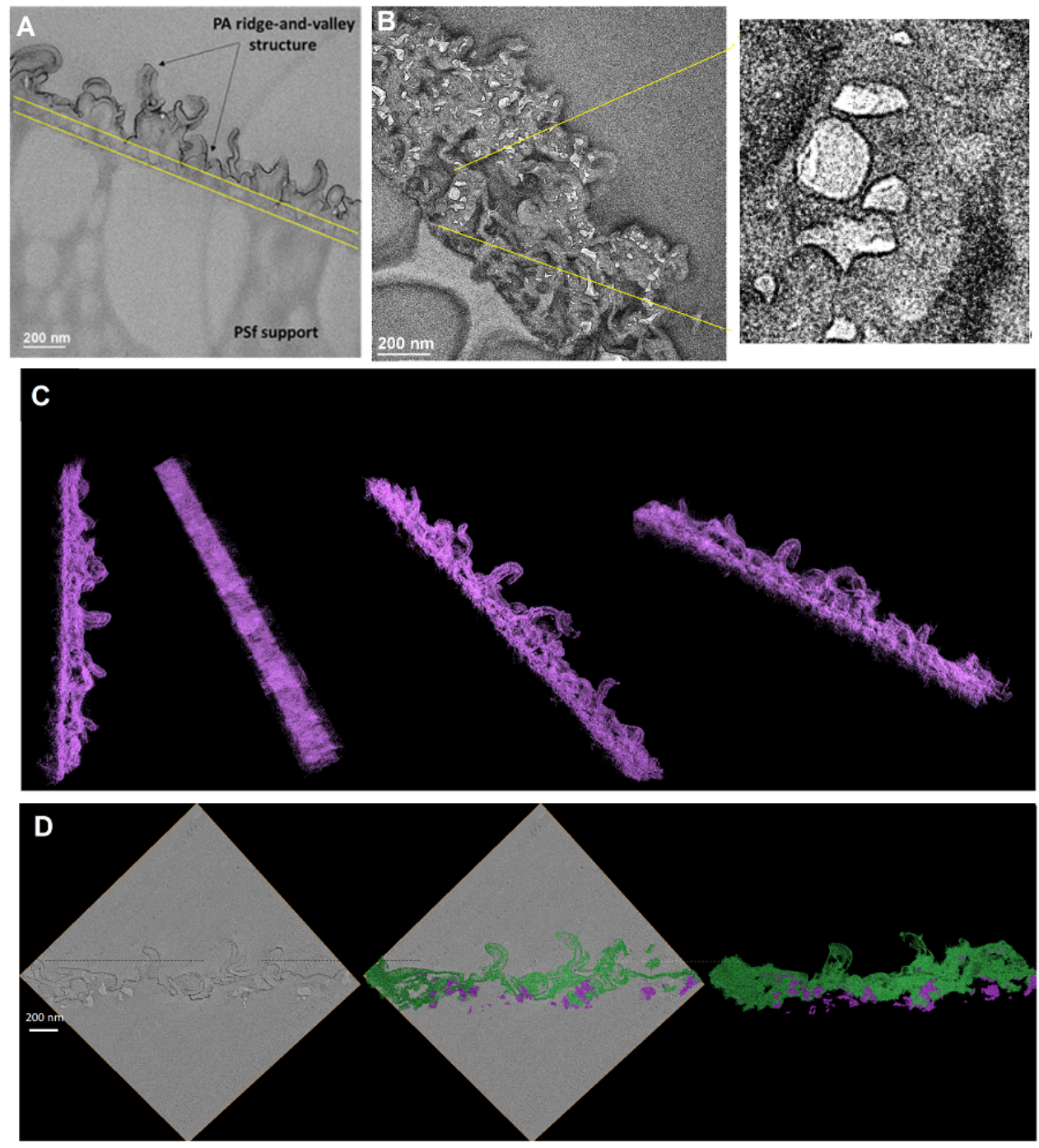

E
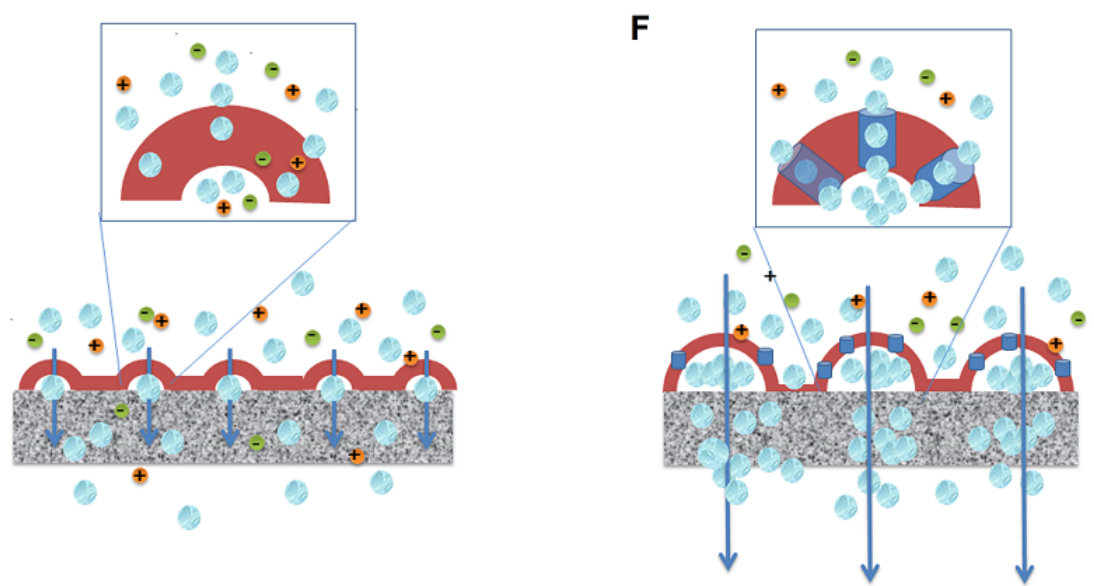

Fig. 2. Morphological TEM characterization of the membranes. TEM cross-sectional images of (A) the pristine PA on porous PSf support and (B) detail of the top layer of the TFC-HC6 membrane with high magnification (membranes stained with $\mathrm{OsO}_{4}$ ). (C) 3D reconstruction TEM tomography of TFC-HC6 membrane in different tilting angles. (D) Instantaneous shots of TFC-HC6 membrane tomography at a fixed angle: the PA layer formed on the surface of the PSf porous support (support not visible) is marked in green. The less dense brighter spots, which could correspond to the AWC/PA nanoparticles, are marked purple and have a relative occupied volume of $20 \%$. Schematic representation of the selective transport of water (blue spheres) versus ions (green and red spheres) across (E) compact pristine TFC and (F) AWC-embedded TFCHC6 membranes with larger surface area and additional water channels. 

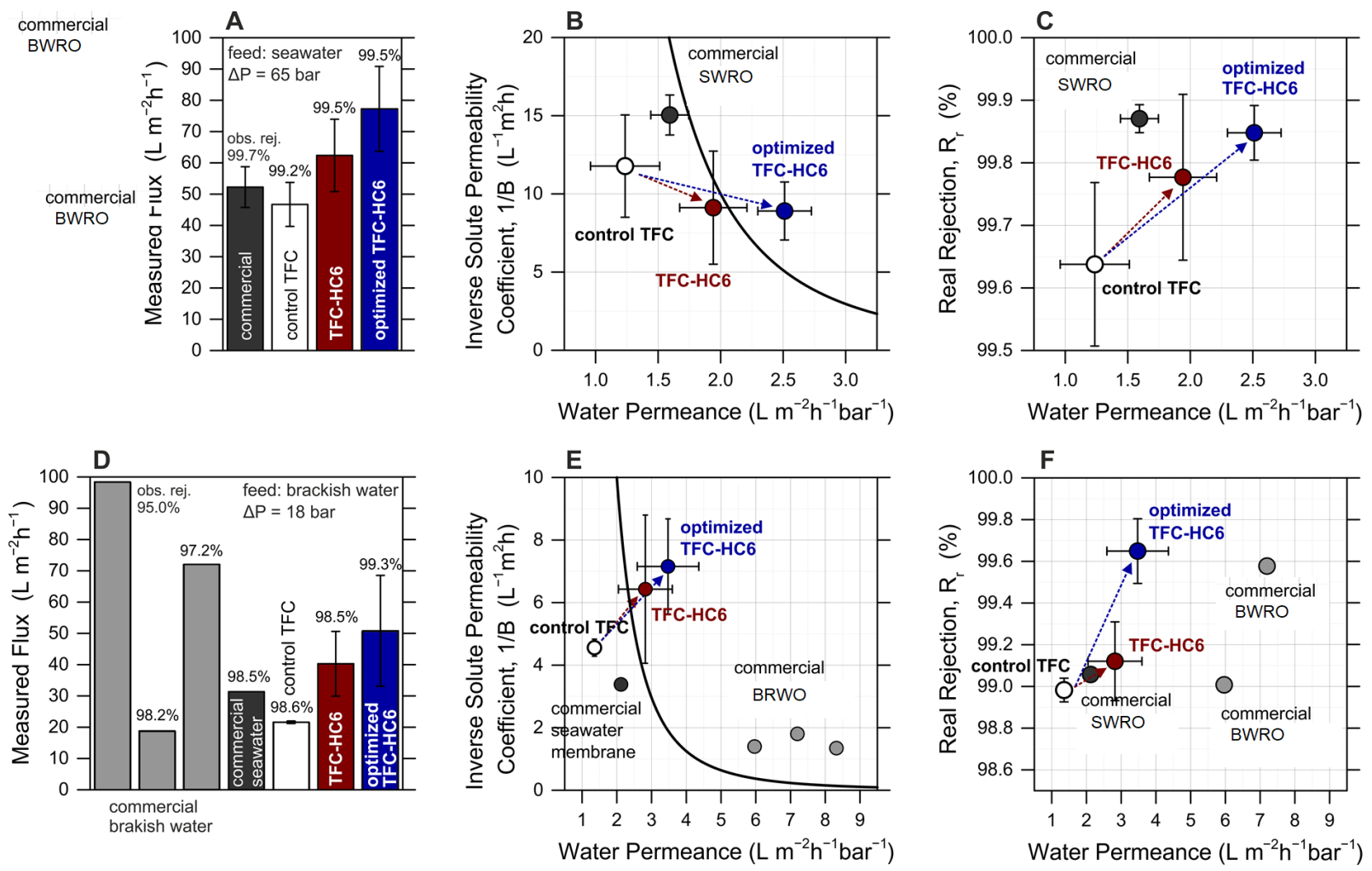

Fig. 3. Performance of the membranes in the desalination of (A-C) seawater and (D-F) brackish water. (A, D) Experimental water flux and observed solute rejection of control TFC membranes, commercial membranes, and TFC-HC6 membranes incorporating AWCs at $0 \%$ recovery. The filtration conditions were: 65 bar applied pressure with $35000 \mathrm{ppm} \mathrm{NaCl}$ feed solution at $\mathrm{pH} 8$ for seawater desalination; 18 bar applied pressure with $5800 \mathrm{ppm} \mathrm{NaCl}$ feed solution at pH 7 for brackish water desalination. (B, E) Selectivity-productivity trade-off graphs plotting the inverse solute permeability coefficient of the membranes as a function of their respective water permeance; the solid line depicts the proposed TFC upper bound relationship. (C, F) Calculated real rejection of the membranes as a function of respective water permeance. The feed solution temperature in all the tests was $27 \pm 1{ }^{\circ} \mathrm{C}$.

Herein, we demonstrate the formation of a new type of PA-based hybrid material with a different complex structure and enhanced water transport properties. The nanostructural distribution of dense and sponge-like PA/AWC regions within the TFC films are essential to control the membrane transport performance, since they determine the pathways for the water flow and diffusion across the active film. Specifically, optimized TFC-HC6 active layers comprising artificial water channels provided a consistent increase in water flux of roughly $75 \%$ and $150 \%$ in the desalination of seawater (Fig. 3A) and brackish water (Fig. 3D), respectively, compared to control TFC membranes that were synthesized without the HC6 molecules. 
This notable productivity was achieved while maintaining or, in many instances, while improving the observed $\mathrm{NaCl}$ solute rejection to values of $\sim 99.5 \%$, relative to control membranes. The membranes proposed in this study also displayed a better combination of productivity and selectivity compared to marketed state-of-the-art membranes obtained by industrially optimized technology. The application of our proposed procedure to these commercial materials would potentially result in the same relative increase in water flux that was observed for lab-made membranes.

A conventional and robust method to compare different membranes is to plot their performance in a permeability-selectivity trade-off chart. Traditional TFC membranes are constrained by an upper bound behavior, as shown in Fig.3B and 3E. While the data associated with lab-made control membranes are placed below the proposed upper bound, mostly due to a low water permeance, the membranes incorporating water channels defeat the trade-off. This result is especially true for optimized TFC-HC6 membranes, whose permeability-selectivity performance places them well beyond the bound line, suggesting that these materials are characterized by a different mechanism of mass transport in distinction to traditional polyamide layers. The improvements accomplished with the incorporation of AWCs are especially evident when the real rejection provided in desalination experiments is plotted against the water permeance (Fig.3C and 3F).

The membranes TFC-HC6 incorporating AWCs were further assessed when applied as seawater desalination filters. Experiments were conducted by achieving 50\% recovery of freshwater from a synthetic feed approximating the salinity and the $\mathrm{pH}$ of typical seawater. The data in Figs. 4A and 4B show the trend of permeate flux as a function of recovery: the flux profile decreases steadily from the initial value of $75 \mathrm{~L} \mathrm{~m}^{-2} \mathrm{~h}^{-1}$ due to the reduction in driving force as the feed solution was increasingly concentrated. The average water flux of the test was $34.4 \mathrm{~L} \mathrm{~m}^{-2} \mathrm{~h}^{-1}$ and the concentration of the product water was $290 \mathrm{mg} / \mathrm{L}$, a value related to an excellent palatability based on the WHO Guidelines for Drinking-Water Quality. This experiment approaches the flux profile and the solute concentrations that would be obtained inside a pressure vessel of a full-scale system for a single pass SWRO process. The enhanced fluid dynamics of real elements would further reduce the effect of 
concentration polarization when compared with lab-scale experiments, thus realizing both larger water fluxes and a higher permeate quality.
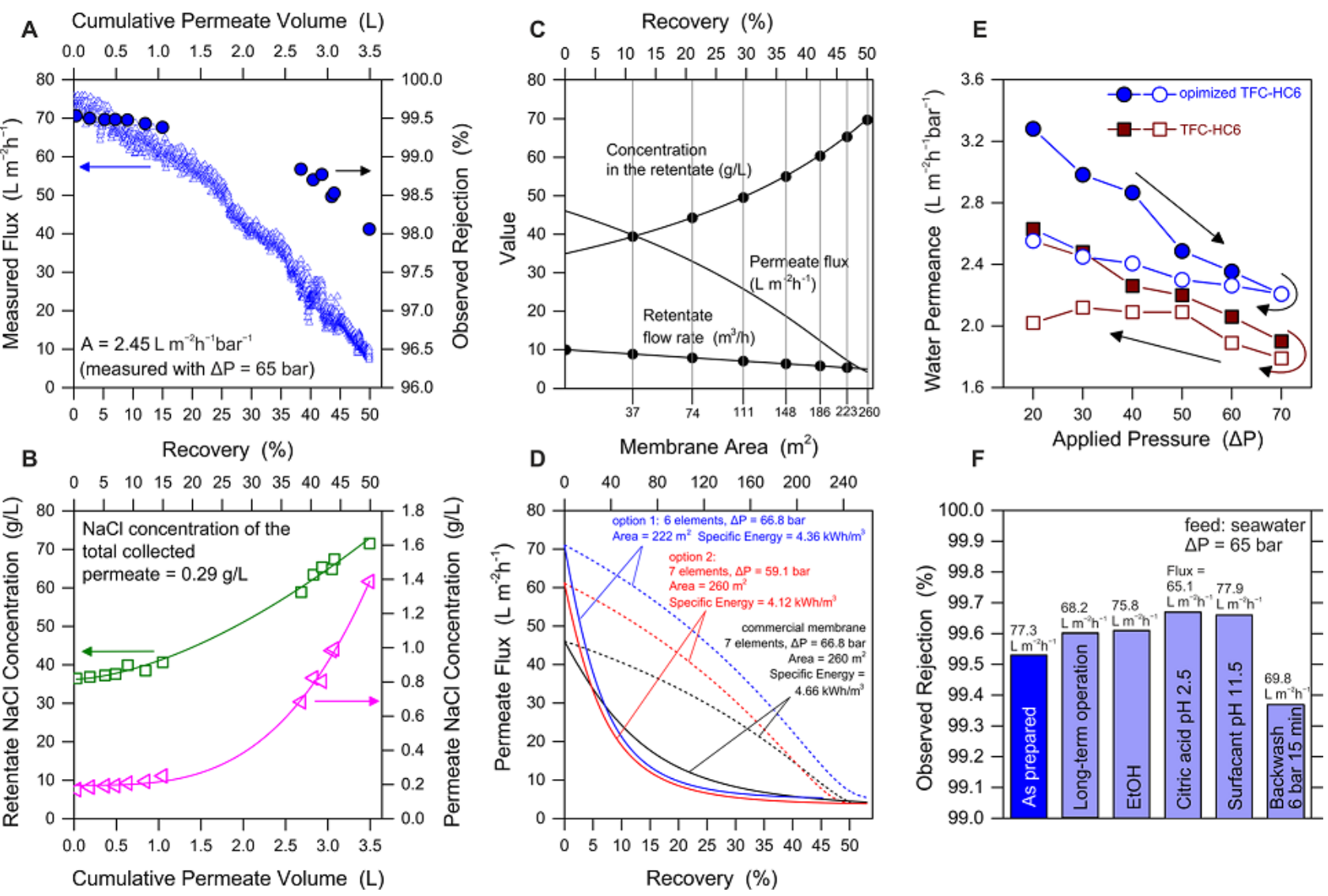

Fig. 4. Application of the membranes in typical seawater desalination operation. (A, B) Experimental water flux, observed solute rejection, feed and permeate stream concentrations as a function of recovery and cumulative permeate volume; here, the filtration conditions were: 65 bar applied pressure with $35000 \mathrm{ppm}$ $\mathrm{NaCl}$ feed solution at $\mathrm{pH} 8$ and a final recovery of 50\%. (C) Validation of the vessel-scale model for SW30XHR commercial membranes and (D) outcome of the application of the model for the commercial membranes and for the TFC-HC6 membranes incorporating AWCs; here, the profile of permeate flux within the vessel is plotted as a function of (solid lines) space, i.e., cumulative membrane area, or (dash lines) recovery rate. (E) Experimental water permeance in tests comprising a stepwise increase followed by a stepwise decrease of applied pressure. (F) Experimental performance of membranes subjected to mechanical or chemical stress; here, the filtration conditions were: 65 bar applied pressure with $35000 \mathrm{ppm} \mathrm{NaCl}$ feed solution at $\mathrm{pH} 8$ and recovery of $0 \%$.

Therefore, a vessel-scale model was implemented to compute the membrane area and the energy required to extract high-quality water from a feed of seawater, by applying current commercial RO membranes and the biomimetic membranes proposed in this work. As shown in Fig.4C, the vesselscale model, represented by solid lines, was first validated with high-rejection SW30XHR-400i elements, against the results provided by the software Wave and plotted as black dots. Once validated, the model was thus applied in two optional implementations of the biomimetic membranes (Fig.4D). 
The modeling outcome suggests that, if the same pressure of 66.8 bar is applied in the vessel, $50 \%$ recovery desalination may be achieved with six instead of seven elements in series, which are instead needed when applying current commercial seawater desalination membranes. In other words, the larger permeance of the membrane TFC-HC6 incorporating AWCs would allow the use of a smaller membrane area, translating in near proportional savings in capital and membrane replacement costs, with also a reduction of the specific energy from 4.66 to $4.36 \mathrm{kWh}$ for each $\mathrm{m}^{3}$ of product freshwater. A possibly more advantageous configuration would involve the same number of elements with respect to current commercial membranes, i.e., identical membrane area and capital costs, but a lower applied pressure of 59.1 bar. According to the model, this arrangement would provide a more uniform permeate flux within the vessel and a noteworthy $12 \%$ reduction of the required energy. It is important to highlight that the application of membrane elements is subject to several constraints in terms of minimum and maximum cross-flow rate, maximum permeate flux, as well as maximum recovery rate. In consequence, the exact real-plant arrangement would have to be appropriately tailored for the characteristics of each membrane type. That being said, our model provides a useful preliminary estimate of the capital and operational savings achievable when applying membranes with higher productivity.

A final set of experiments were conducted to understand the resilience of the TFC-HC6 layers incorporating AWCs to mechanical and chemical stress. The materials displayed a plastic behavior, as membranes could not recover their original permeance once subjected to high hydraulic pressures; see Fig.4E. This phenomenon needs further investigation, but appears to be to some degree linked to the presence of the AWCs, as it was significantly less pronounced for control TFC layers (not shown). Nevertheless, the novel membranes were notably robust and able to provide fluxes and rejections within experimental error after being exposed to relatively harsh environments or mechanical solicitations. The observations imply their feasible deployment in real systems where they would undergo cycles of physical and chemical cleaning, often with the use of acidic, basic, and/or 
amphiphilic compounds. Perhaps the most significant outcome is related to the ability of the materials to maintain high performance, following immersion in pure ethanol, the most favorable solvent for the HC6 compound. This result suggests that the AWCs were effectively incorporated within the PA matrix and no leaching was detected. The membranes remained stable, and practically the same permeance and rejection were observed (Fig. 4F). This is actually what ultimately counts, considering the membrane application.

This rationalization corroborates the results discussed above; suggesting that the embedded selfassembled nanoparticles did not generate defects of the uppermost active layer. Indeed, the highly selective AWC are intrinsically promoting the preferential selective passage of water through the nanoparticles together with a significantly better perm-selective behavior through a selective layer with larger surface area. It also implies an excellent structural compatibility of AWCs with the surrounding PA matrix, a necessary requisite to construct a seamless active layer without defects.

1. M. Elimelech, W. A. Phillip, Science 333, 712-717 (2011).

2. P. J. J. Alvarez, C.K. Chan, M. Elimelech, N. J. Halas, D. Villagran, Nature Nanotech. 13, 634$641(2018)$.

3. M. A. Shannon P. W. Bohn, M. Elimelech, J. G. Georgiadis, B. J. Marinas, A. M. Mayes, Nature 425, 301-310 (2008).

4. J. E. Cadotte, R. J Petersen, R. E. Larson, E. E Erikson, Desalination 32, 25-31 (1980).

5. S. Karan, Z. Jiang, A. G. Livingston, Science 348, 1347-1351 (2015).

6. M. R. Chowdhury, J. Steffes, B. D. Huey, J. R. McCutcheon, Science 361, 682-686 (2018).

7. Z. Tan, S. Chen, X. Peng. L. Zhang, C. Gao, Science 360, 518-521 (2018).

8. B.-H. Jeong, E. M. V. Hoek, Y. Yan, A. Subramani, X. Huang, G. Hurwitz, A. K. Ghosh, A. Jawor, J. Membr. Sci. 294, 1-7 (2007)

9. J. Duan, Y. Pan, F. Pacheco, E. Litwiller, Z. Lai, I. Pinnau, J. Membr. Sci. 476, 303-310 (2015) 
10. T. V. Ratto, J. K. Holt, A. W. Szmodis, Membranes with embedded nanotubes for selective permeability, US Patent 7993524 (2011).

11. J. R. Weber, A. Deshmukh, M. Elimelech, Environ. Sci. Technol. Lett. 3, 112-120 (2016).

12. P. Agre, Angew. Chem. Int. Ed. 43, 4278-4290 (2004)

13. U. K. Eriksson, G. Fischer, R. Friemann, G. Enkavi, E.Tajkhorshid, R. Neutze, Science 340, 1346-1349 (2013).

14. C. Y. Tang, Y. Zhao, R. Wang, C. Hélix-Nielsen, A. G. Fane Desalination, 308, 34-40 (2013)

15. M. Barboiu, Angew. Chem. Int. Ed. 51, 11674-11676. (2012).

16. M. Barboiu, A. Gilles, Acc. Chem. Res. 46, 2814-2823 (2013).

17. Y. Le Duc, M. Michau, A. Gilles, V. Gence, Y.-M. Legrand, A. van der Lee, S. Tingry, M. Barboiu, Angew. Chem. Int. Ed., 50, 11366-11372 (2011)

18. X. B. Hu, Z. Chen, G. Tang, J. L. Hou, Z. T. Li J. Am. Chem. Soc., 134, 8384-8387. (2012).

19. W. Si, L. Chen, X.-B. Hu, G. Tang, J. L. Hou, Z. T. Li, Angew. Chem. Int. Ed., 50, 12564-12568 (2011).

20. E. Licsandru, I. Kocsis, Y.-x. Shen, S. Murail, Y.-M. Legrand, A. van der Lee, D. Tsai, M. Baaden, M. Kumar, M. Barboiu, J. Am. Chem. Soc. 138, 5403-5409 (2016).

21. R. H. Tunuguntla, R. Y. Henley, Y.-C. Yao, T. A. Pham, M. Wanunu, A. Noy Science 357, 792796 (2017).

22. I. Kocsis, M. Sorci, H. Vanselous, S. Murail, S. E. Sanders, E. Licsandru, Y.-M. Legrand, A. van der Lee, M.Baaden, P. B. Petersen, G. Belfort, M. Barboiu, Science Adv., 4, eaao5603 (2018).

23. J. R. Weber, M. Elimelech, Science Adv., 4, eaar8266 (2018).

24. R. L. McGinnis, K. Reimund, J. Ren, L. Xia, M. R. Chowdhury, X. Sun, M. Abril, J. D. Moon, M. M. Merrick, J. Park, K. A. Stevens, J. R. McCutcheon, B. D. Freeman Science Adv., 4, e1700938 (2018).

25. B. Mi, Science 343, 740 (2014). 
26. Y. Yang, X. Yang, L. Liang, Y. Gao, H. Cheng, X. Li, M. Zou, A. Cao, R. Ma, Q. Yuan, X. Duan, Science 364, 1057-1062 (2019).

27. Y.-x. Shen, W. C. Song, D. R. Barden, T. Ren, C.Lang, H. Feroz, C. B. Henderson, P.O. Saboe, D. Tsai, H. Yan, P. J. Butler, G. C. Bazan, W. A. Phillip, R. J. Hickey, P. S. Cremer, H. Vashisth, M. Kumar, Nature Commun. 9, 2294 (2018).

28. L. Lin, R. Lopez, G. Z. Ramon, O. Coronell, J. Membr. Sci., 497, 365-376 (2016).

29. F. Pacheco, R. S., M. Reinhard, J.O. Leckie, I. Pinnau, J. Membr. Sci. 501, 33-44 (2016).

30. M. C. Y. Wong, L. Lin, O. Coronell, E. M. V. Hoek, G. Z. Ramon, J. Membr. Sci. 500, 124-135, (2016).

31. Y. Li, M. M. Klosowski, C. M. McGilvery, A. E. Porter, A. G. Livingston, J. Membr. Sci. 534, 9-17 (2017). 